\title{
Performance Analysis of RPL Routing Protocol for WBANs
}

\author{
Prof. Prachiti Deshpande \\ Professor, Department of Computer Engineering, B.D.C.E Wardha India \\ prachitideshpande@gmail.com
}

\begin{tabular}{|c|c|}
\hline Article History & Abstract \\
\hline $\begin{array}{l}\text { Article Submission } \\
19 \text { November } 2015 \\
\text { Revised Submission } \\
17 \text { January } 2016 \\
\text { Article Accepted } \\
1 \text { March } 2016 \\
\text { Article Published } \\
31^{\text {st }} \text { March } 2016\end{array}$ & $\begin{array}{l}\text { This research article focuses on evaluating the performance of RPL protocol for } \\
\text { WBANs. The WBANs place stringent requirements for the protocol design due to its } \\
\text { adhoc nature, heterogeneous data rate, requirement of mobility and ultra-low power } \\
\text { consumption. Many works have been focused on evaluating the RPL protocol through } \\
\text { simulation; on the other hand the real world implementation has its own } \\
\text { vulnerabilities. So it is important that the protocol should be experimented on the } \\
\text { real hardware devices. This work aims in developing an inter-WBAN to monitor the } \\
\text { vital health parameters such as heart beat and body moisture level of two or more } \\
\text { patients simultaneously. It has been proved that the RPL performance is far superior } \\
\text { to simple multi hop technique by comparing various performance attributes. } \\
\text { Keywords: WBAN;RPL; Patient monitoring; contiki }\end{array}$ \\
\hline
\end{tabular}

\section{Introduction}

The Internet Engineering Task Force (IETF) is the standardization unit of protocol suites. The LLN comprises of constrained nodes [1]. In most of cases they are either operated through battery or energy forage. The unique characteristics of the LLNs led to the formation of the ROLL working group and it belongs to the Routing Area Group (RTG). The routing is a fundamental piece to accomplish the task of Internet of Things. The protocol that addresses all the requirements of LLNs was finally created by IETF called RPL [2]. Application areas of LLN include automation sector, healthcare systems and various WSNs [3]. The ROLL Working Group has developed applications specific to routing requirements.

A WBAN find its importance in various medical sensors that are fixed inside and outside of the human body. The demand for an effective communication technology in the health care sector is greater than before. The technology should reduce the complexity involved in remote monitoring of patients. The sensor nodes are power constrained devices. The links tend to be lossy because of its wireless nature. The number of nodes in intra WBAN is limited to single human being; it will get increased when constructing inter-WBAN. Also the area covered by the network is huge while comparing with intra-WBAN. To overcome the performance degradation of the network a robust routing protocol has to be introduced. The main objective of the work is to develop a wireless inter body area network that continuously monitor the human health parameters and reports the information to the nearby PC/sinks by implementing RPL. The rest of the paper is organized into four sections. The section brings the picture of IPV6 routing with RPL and third section describes the significant components of medical monitoring. The fourth section presents the proposed system design and implementation. The Results and discussion section analyzes the performance of the proposed design with the two different routing metric. The final section concludes our work with useful remarks.

\section{Related IPV6 Routing with RPL}

There are wide range of equipment such as smart phones, cars, weather sensors and cardiac monitor etc. More than billions of these devices have to be connected to the internet. They are not necessarily a component of a Personal Area Network. The connected networks are known as low power and lossy networks and the possible IPv6 routing protocol to connect these devices to the internet is RPL (pronounced as Ripple). RPL is a distance 
vector routing protocol that forms a Destination Oriented Direct Acyclic Graph (DODAG) to its root [10]. The distance vector routing is simple routing protocol hence it is very much suitable for the resource constrained devices. The RPL requires bidirectional links to check the reachability. After verifying the links, it will transmit the messages. The routing metric can be based on the nodes or the link reliability. ETX variations are averaged /filtered to be stable enough for route computation [13]. To calculate the best path the routing metric has to be optimized with respect to several constrains such as throughput, latency etc. The task is accomplished with the help of objective function. Three basic mechanisms involved in RPL are explained below.

\section{A. DAG Construction}

The idea of DAG helps RPL to route packets properly to the sink. Otherwise the packets will keep flowing in circles and never end up at the desired destination. Let Routing metric to be expected transmission (ETX) count. The Objective is to minimize the ETX. The Depth of a node is ETX to sink. The sink or border router multicasts Direct acyclic graph Identification Object. Neighbor nodes receive and process DIO packets[15]. Neighbor nodes add sink as a DAG (Direct Acyclic Graph) parent considering link metrics and optimization objective. Children node's depth are calculated (depth ETX) from sink. When the trickle timer expired the nodes will start broadcasting DIO packets in return. The Level one children can add another level one child as alternate DAG parent if depth is less than or equal to the direct link depth. This process continues until timer on each node in the network gets expired and thus DAG is formed.

\section{B. Destination Advertisement}

Destination Advertisement builds up routing table starting from leaf nodes to sink/router. Destination advertisement can be triggered either by the DAG root or the node that detects a change. Sink triggers destination advertisement mechanism through DIO and made the packet to flow towards the leaf nodes. Leaf nodes emit network address to their immediate parent with DAO (Destination advertisement object) indicating reachability. The parent will store the prefix address of the leaf nodes and emits network address to its immediate parent with DAO indicating reachability. This process will continue till it reaches sink.

\section{DAG Maintenance}

When a link between two nodes in a network goes bad, the link will be terminated. Thus the lower part of the DAG will get separated from the upper DAG and get floating without proper ground to root. Floating part of the DAG has no parent and thus the top node will become the root of its own floating DAG. To inform about the change floating DAG's root will multicast DIO. If any of the sub DAG has an alternate DAG parent grounded to sink, it will terminate the connection from the floating DAG's root, else it will remain as a floating DAG until it receives a DIO packet from any of the nodes that are present in the grounded DAG.

\section{Proposed WBAN Monitoring Vital Health Parameters}

Monitoring is the observation of an event.It is done by continuously measuring certain vital health parameters. The major hardware used in the work is the TelosB or Tmote Sky mote. Here the motes are being programmed using a real time operating system called CONTIKI OS[17]. The major components of medical monitoring are biosensor, translating component, display device and communication link.

Sensors are the medical monitors which includes bio and mechanical sensors. It can be interfaced externally with the motes using the expansion connectors. In this work the widely employed sensors are the heart beat monitoring sensors which is used to monitor the heartbeat of the patient and moisture sensor to check whether the patient is sweating and PIR (Passive Infra-Red) sensor to detect the movement. The mote with sensor is termed as node.Heart beat sensoris designed to give digital output signals of heat beat when a finger touches it. The TELOSB mote with heartbeat sensor is shown in the Figure 1. 


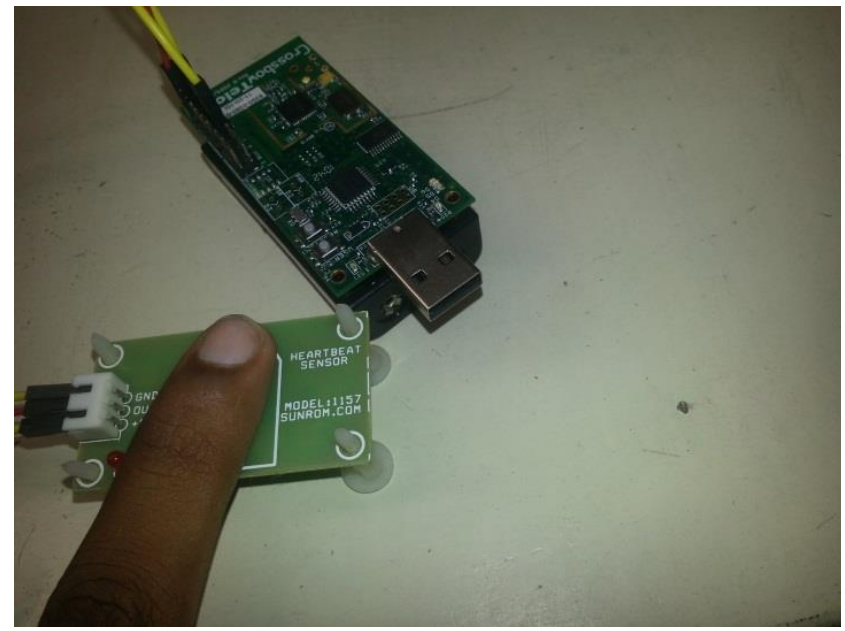

Fig 1: TelosB mote with Heartbeat sensor

Liquid/Moisture sensor detects liquid or moisture between leads and gives low level output. These wires when they detect moisture, the circuit becomes closed and provides a conducting path. TelosB mote with Moisture sensor is shown in the Figure 2.

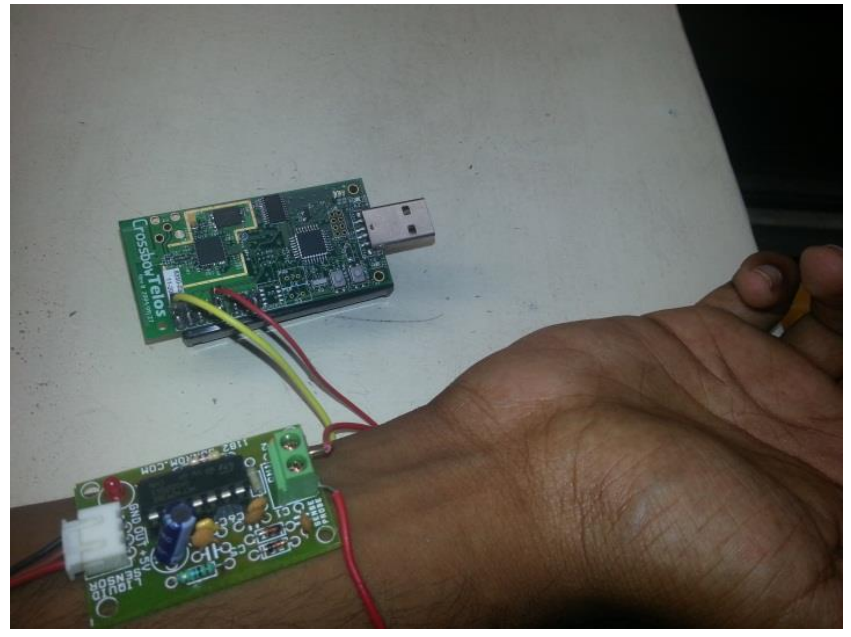

Fig 2: TelosB mote with Moisture sensor

The translating component is responsible for converting the sensor information to machine understandable format. The operation will be performed by the peripherals connected to the sensor.The physiological data is displayed continuously on display devices (like CRT, LED OR LCD SCREEN) Here the sink node is connected to a doctor PC or medical server serves as a display device. The TELOSB/Tmote Sky has IEEE 802.15.4 compliant chipcon cc2420 radio for wireless communication. This radio is controlled by MSP430 Microcontroller.

There are three kinds of nodes used in the whole network namely source, intermediate node and sink. The first kind of node is connected to the sensor which is in turn connected to the body of the patient. This node collects the information from sensor and sends it to the server/sink through the route created by RPL. The second kind of node acts as a routing node. With the help of this routing table data packets are routed properly to the main server. This results in a multi-hop network where the data is transferred from one mote to another mote. The number of intermediate motes can be increased depends upon the distance to be covered. The node which is connected to the PC serves as a sink and displays the information about the patient on the computer screen. The flowchart which explains the monitoring of vital health parameters is depicted in the Figure 3. 


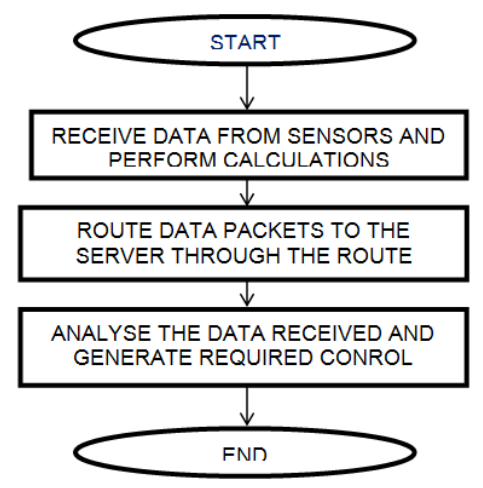

Fig 3: Flow chart - Working of Inter WBAN

\section{Implementation of RPL Protocol in WBAN}

The entire setup consists of seven telosB motes and biosensors that are interfaced with the patient whose conditions are to be monitored. With the help of microcontroller, motes perform calculations and keep posting about the patient's health continuously. The inter WBAN communication is shown in the Figure 4 In case of a single patient the distance between any two nodes is in the range of centimeters it will not consume more power when they follow a fixed path to server. But multiple patients with associated mobility will result in more consumption of power, improper end to end delay and reduced throughput. The RPL DAG construction and maintenance provides solution to the above mentioned problems. This is shown in figure 4.

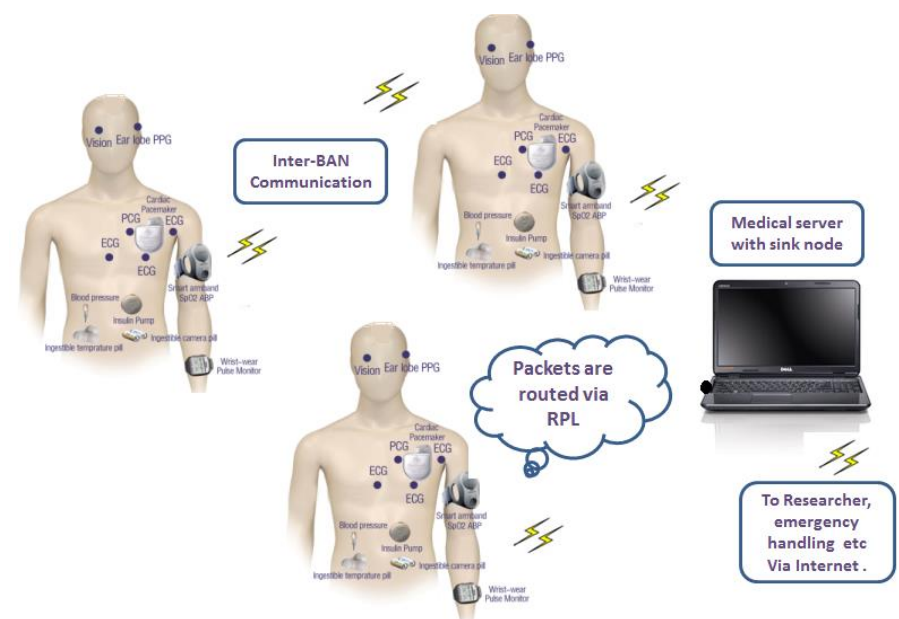

Fig 4: Inter WBAN communication

In order to implement RPL in inter WBAN using TELOSB motes, a simple UDP communication was considered. All the motes except one are loaded with udp-sender program, which gets data from the biosensors and route the udp packets wirelessly through routing nodes. One TELOSB mote loaded with udp-sink program, which collects the information from different patients and displays it in a monitor. Contiki system uses concurrent programming, which means two or more processes are executed simultaneously. UDP sender program consists of three processes. First process helps in constructing DAG, establishing connection between sender and sink. Second process helps in collecting data from the sensor by periodically reading the input ports that are connected to the sensors. For proper working of heart beat, sensor should sense the input ports at every $300 \mathrm{~ms}$. Third process is used for timing related activities. After waiting for few seconds, third process will post the time value from clock to the second process since the second process helps in keeping count of the heart beat/digital signal from sensor. Other sensors like moisture detector and motion detector doesn't need third process. Whenever it detects a change it will pass 
the data to process one. The first process creates a UDP packet and route the packet through the route dictated by RPL. Sink node receives the packet and displays the data in the screen. The implemented WBAN is shown in the Figure 5. The implementation is done with two different routing metrics namely ETX and Minimum hop route. By default conikiRPL uses ETX (Expected number of transmissions for successful delivery of data) as routing metric. The ETX has been replaced by minimum number of hops which is defined in the objective function 0[18]. The ETX can be taken into consideration in situations where power consumption is a vital factor, i.e., in places such private hospitals, well financed medical centers, etc... In scenarios where there is a constraint in the number of nodes used, the hops count parameter can be taken for the path calculation, i.e., in places such as military encampments, remote locations where archaeological surveys are held, etc...

\section{Simulation Results}

The performance evaluation is done using Cooja simulator to ensure the integrity of the code before uploading it to the hardware. The Simulator is also used to analyze various parameters involved in the network and the results and their inferences are described. An analysis has been done by considering routing of data with two different routing metrics namely ETX and Minimum hop route. Figure 5 shows the DAG structure formation in ETX routing metric network and Fig 6 shows that the network structure of hops.

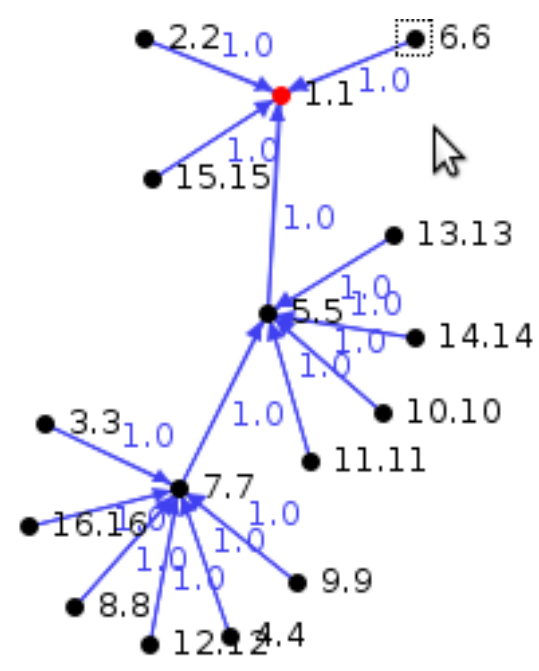

Fig 5: Network structure (ETX)

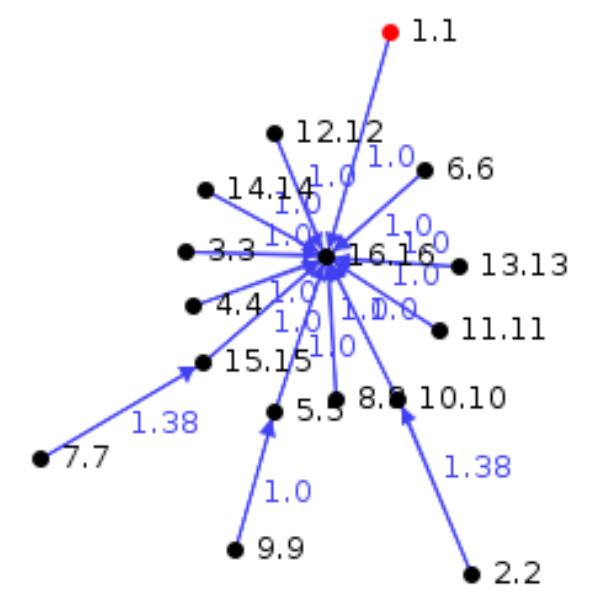

Fig 6: Network structure (Hops) 
TABLE 1: Power Consumption - ETX Routing Metric

\begin{tabular}{|c|l|l|l|l|l|}
\hline $\begin{array}{l}\text { Number } \\
\text { Nodes }\end{array}$ & $\begin{array}{l}\text { CPU } \\
\text { authority } \\
(\mathbf{m w})\end{array}$ & $\begin{array}{l}\text { LPM } \\
\text { authority } \\
(\mathbf{m w})\end{array}$ & $\begin{array}{l}\text { Listen } \\
\text { authority } \\
(\mathbf{m w})\end{array}$ & $\begin{array}{l}\text { Transmit Power } \\
(\mathbf{m w})\end{array}$ & $\begin{array}{l}\text { Total } \\
\text { Power } \\
(\mathbf{m w})\end{array}$ \\
\hline 5 & 0.074 & 0.161 & 0.096 & 0.412 & 0.744 \\
\hline 10 & 0.087 & 0.161 & 0.453 & 0.100 & 0.801 \\
\hline 15 & 0.081 & 0.161 & 0.097 & 0.486 & 0.825 \\
\hline 30 & 0.087 & 0.161 & 0.094 & 0.583 & 0.925 \\
\hline 50 & 0.140 & 0.158 & 1.143 & 0.212 & 1.653 \\
\hline
\end{tabular}

TABLE 2: Power Consumption - Hops Routing Metric

\begin{tabular}{|l|l|l|l|l|l|}
\hline $\begin{array}{l}\text { Number } \\
\text { Nodes }\end{array}$ & $\begin{array}{l}\text { CPU } \\
\text { authority } \\
(\mathbf{m w})\end{array}$ & $\begin{array}{l}\text { LPM } \\
\text { authority } \\
(\mathbf{m w})\end{array}$ & $\begin{array}{l}\text { Listen } \\
\text { authority } \\
(\mathbf{m w})\end{array}$ & $\begin{array}{l}\text { Transmit Power } \\
(\mathbf{m w})\end{array}$ & $\begin{array}{l}\text { Total } \\
\text { Power } \\
(\mathbf{m w})\end{array}$ \\
\hline 5 & 0.074 & 0.161 & 0.109 & 0.403 & 0.748 \\
\hline 10 & 0.090 & 0.161 & 0.461 & 0.097 & 0.808 \\
\hline 15 & 0.077 & 0.161 & 0.447 & 0.168 & 0.854 \\
\hline 30 & 0.089 & 0.161 & 0.544 & 0.181 & 0.975 \\
\hline 50 & 0.267 & 0.155 & 1.491 & 0.319 & 2.232 \\
\hline
\end{tabular}

The total power consumed by the nodes using Etx and Hop routing metric is tabulated in Table I and II. CPU refers to the power consumption during the full power mode, LPM refers to the low power mode, transmit is for the transmit operations, and listen for the listening (e.g. packet capture) operations. Power consumed by CPU and LPM are very less compared to transmit power and listen power. The Figure .8 shows that effect of routing metric on power consumption. From the Figure 8 it is verified that power consumed by network constructed with HOPS metric is higher compared to the network constructed with ETX metric. This is because ETX constructs path with minimum expected number of transmission irrespective of number hops required to reach the server/sink, whereas HOPS constructs path with minimum number of hops irrespective of the ETX values of the links. So, while considering path with less number of hops and very high value of ETX, power consumption will be much higher.

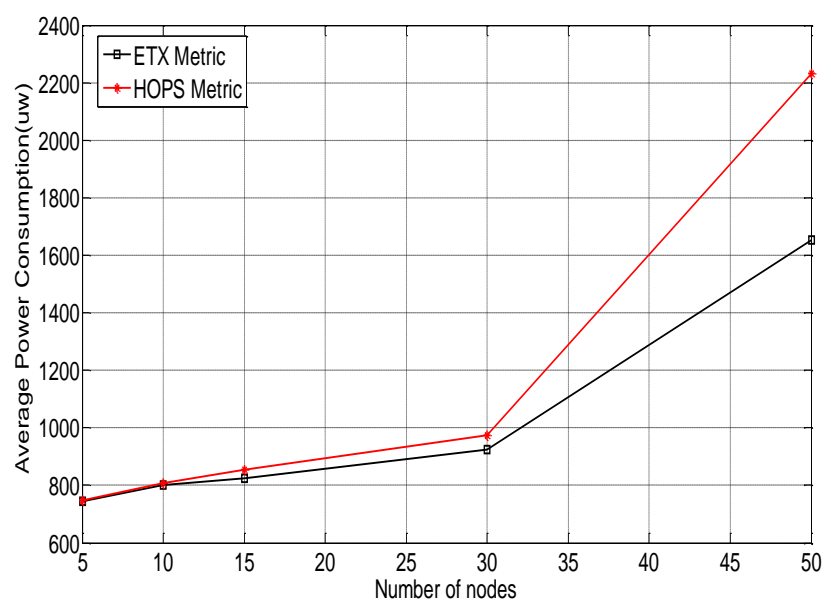

Fig 7: Effect of Routing Metric on Power consumption 
The effect of routing Metric on end to end delay is shown in Figure .9.It is clear from graph that average end to end delay is greater for HOP metric than ETX metric. While choosing path with greater ETX value there will be huge amount of packet loss and retransmission which results in increased end to end delay. This is the reason for reduced throughput too. The throughput of the network when the number of node increased is revealed in Figure .10. When number of nodes increases; all the available channels are utilized by more number of nodes. The data traffic will increase which in turn will decrease the throughput. Number of nodes required to monitor ECG of a patient is ten and it requires a data rate of 600 bps for proper working [8]. Besides heart beat rate monitoring and moisture detection, it is also possible to monitor ECG of the patient using this system when the numbers of nodes are limited. In [8], it is also mentioned that data rate required for blood flow, respiratory, blood pressure, blood $\mathrm{pH}$ and body temperature are $0.48,0.24,1.2$, 0.048 and 0.0024 bps respectively which are also all in the acceptable range.

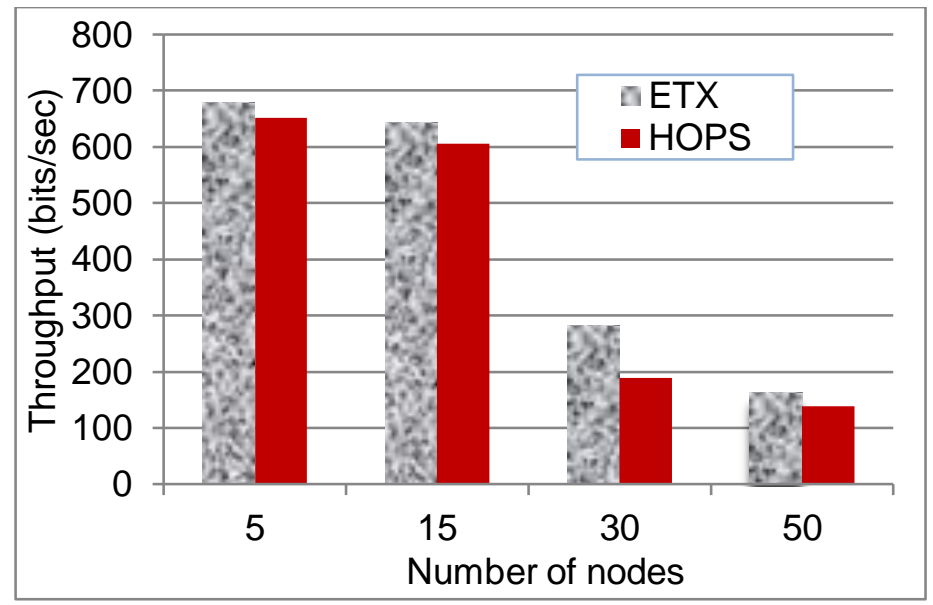

Fig 8: Throughput analysis - ETX Vs HOPS

Every source node in the network is made to transmit packet every second. When the number of source nodes in the network is five, only five packets will be transmitted in a second. Since the data traffic is low packets received will be almost equal to packets transmitted. But when number of nodes increases in the network, data traffic will also increase considerably resulting in much more collision than fewer nodes network.From the Table.III it is confirmed that packet loss is more in HOP metric compared to ETX metric. This is because while constructing a network with HOP metric, always chooses path with minimum no of hops. ETX value is inversely proportional to quality of the link. A link which has low value of ETX has very good quality and thus packet loss will be very small. Since HOP metric chooses poor quality link to minimize number of hops, it will always have poor packet loss ratio.

TABLE 3: Packet Loss Analysis

\begin{tabular}{|c|c|c|c|}
\hline $\begin{array}{c}\text { Number of } \\
\text { nodes }\end{array}$ & $\begin{array}{c}\text { Average No. of packets } \\
\text { transmitted per sec }\end{array}$ & $\begin{array}{c}\text { Average No. of packets } \\
\text { received per sec (etx) }\end{array}$ & $\begin{array}{c}\text { Average Number of packets } \\
\text { received per sec (hops) }\end{array}$ \\
\hline 5 & 5 & 4.99933 & 4.676 \\
\hline 15 & 15 & 14.9446 & 13.977 \\
\hline 30 & 30 & 29.902 & 28.758 \\
\hline 50 & 50 & 49.174 & 48.208 \\
\hline
\end{tabular}

\section{Conclusion}

An inter-wireless body area network, which monitors the health of patients continuously, has been implemented by using TELOSB motes and Contiki RTOS. Useful medical data like heart-beat, moisture level and movement abnormality of the patients are monitored with the help of heart-beat sensor and liquid Moisture sensor. The sensed data are transmitted wirelessly through the route defined by the routing protocol RPL. Performance of the network is analyzed with different routing metrics like Expected number of transmissions (ETX) and Minimum number of hops (HOPS) using cooja simulator in detail. RPL forms paths towards roots based on one 
or more metrics, from the analysis it is shown that RPL is suitable protocol for WBAN in terms of throughput, power and packet loss. From the evaluation it is concluded that increase in number of nodes gradually increase the end to end delay, which is not acceptable in most of the medical conditions hence the future work would be to design a efficient routing metric exclusively meet the requirements of the body area network.

\section{References}

[1] Jean Philippe Vasseur, AdamDunkels. Interconnecting smart objects with IP,The Next Internet , Morgan Kaufmann 2010 Elsevier.

[2] Delphine Christin, Andreas Reinhardt, Parag S. Mogre, Ralf Steinmetz wireless sensor networks and internet of things:selected challenges pp-31-33.

[3] Thomas Clausen, Ulrich Herberg, Multipoint-to-point and broadcast in RPL, Laboratoired 'Informatique (LIX) - EcolePolytechnique, France, 2009.

[4] Kevin C. Lee,Raghuram Sudhaakar, Jianxia Ning, Lillian Dai, Sateesh Addepalli, J. P. Vasseur,andMario Gerla “A Comprehensive Evaluation of RPL under Mobility” International Journal of Vehicular Technology Volume(2012). doi:10.1155/2012/904308

[5] Jonathan Hui, David Culler, SamitaChakrabarti "6LoWPAN: Incorporating IEEE 802.15.4 into the IP architecture”, IPSO alliance, January 2009. [7]BogdanPavkovic, FabriceTheoleyre, AndrzejDuda, Multipath Opportunistic RPL Routing over IEEE 802.15.4, MSWiM'11, October 31-November 4, 2011, Miami DOI -10.1145/2068897.2068929

[6] G. Montenegro, N. Kushalnagar, J. Hui, and D. Culler, Transmission of IPv6 packets over IEEE 802.15.4 networks, Internet proposed standard RFC 4944, Sept. 2007

[7] J. Tripathi, J. de Oliveira, and J. Vasseur, Performance evaluation of routing protocol for low Power and lossy Networks (RPL), 44th Annual Conference on Information Sciences and Systems (CISS), 2010 , pp1-6

[8] Thomas Clausen, Ulrich Herberg, Matthias Philipp, “A Crictical Evaluation of the IPv6 Routing Protocol for Low Power and Lossy Networks", Insitut national de recherché en informatique en automatique, May 2011.

[9] M. U. H. Al Rasyid, D. Prasetyo, I. U. Nadhori and A. H. Alasiry, "Mobile monitoring of muscular strain sensor based on Wireless Body Area Network," 2015 International Electronics Symposium (IES), Surabaya, 2015, pp. 284-287, doi: 10.1109/ELECSYM.2015.7380856.

[10] S. Liu, K. Wang, J. Guo, Y. Wang and X. Qi, "Review on MAC Protocols in Energy-Harvesting Wireless Body Area Networks," 2015 International Conference on Identification, Information, and Knowledge in the Internet of Things (IIKI), Beijing, 2015, pp. 303-304, doi: 10.1109/IIKI.2015.72. 